\title{
Dispersion-Compensating Optical Digital Filters for 40-Gb/s Metro Add-Drop Applications
}

\author{
Jason (Jyehong) Chen, Member, IEEE
}

\begin{abstract}
Optical digital filters that employ optical delay lines are proposed. Properly locating the zeros in the transfer function is shown to enable interleaver pairs to be designed with the same amplitude responses but opposite phase responses. In metro add-drop applications, the interleaver pair can provide up to $50 \%$ of the adding and dropping of the total traffic while simultaneously reducing the insertion loss of the express channel. Both experiments and Monte Carlo simulations demonstrated that the cascaded dispersion of seven pairs of interleavers within the $+/-30-\mathbf{G H z}$ passband is less than $1 \mathrm{ps}$. A total dispersion near zero considerably increases the effective bandwidth and greatly increases the number of cascadable add-drop nodes.
\end{abstract}

Index Terms-Birefringence, metropolitan area network, optical fiber communication, optical fiber devices.

\section{INTRODUCTION}

$\mathbf{E}$ LECTRONIC digital filters, or discrete time filters [1], which employ electrical delay lines, are extensively used in digital electronic circuits. Borrowing the concept from the field of electronics, optical engineers have used optical delay lines to generate optical filter functions. Depending on whether the transfer function has poles, an optical digital filter can be classified as a finite impulse response (FIR) filter and an infinite impulse response filter [1]-[3]. FIR filters can be designed to have the same amplitude responses but have the opposite phase responses by properly locating the zeros in the transfer functions [1], [3]. Most optical digital filters include optical delay lines, directional couplers, and phase shifters. Their applications include (de)multiplexing [4]-[6], adding-dropping [7], equalizing gain [8], and compensating for dispersion [9].

Dense wavelength-division-multiplexing (DWDM) optical systems depend on good optical filters to meet the strict requirements of amplitude response; the ideal filters for such applications are perfectly rectangular filters. The phase response of such filters is equally important in attaining high bandwidth utilization; the ideal filter has a perfectly linear phase response and, therefore, a constant delay without dispersion.

Many DWDM systems use interleavers as multiplexers and demultiplexers. In a metro system, a pair of interleavers can be used in add-drop applications to provide up to 50\% adding and dropping of total traffic while simultaneously reducing the

Manuscript received October 7, 2003; revised January 9, 2004. This work was supported by the National Science Council of the Republic of China, Taiwan under Contract NSC 92-2215-E-009-064.

The author is with the Institute of Electro-Optical Engineering, National Chiao-Tung University, Hsin-Chu 300, Taiwan, R.O.C. (e-mail: jchen@mail.nctu.edu.tw).

Digital Object Identifier 10.1109/LPT.2004.826061

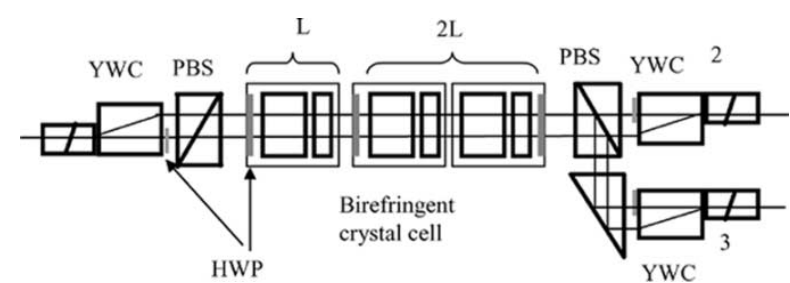

Fig. 1. Possible configuration of an $L-2 L$ interleaver. PBS: polarization-beam splitter. YWC: $\mathrm{YVO}_{4}$ walkoff crystal. HWP: half-wave plate.

insertion loss associated with the express channels. In such an application, the two factors that limit the maximum number of cascadable nodes are the passband (amplitude response) and the group delay (phase response). An interleaver with a wide passband and a linear phase response is preferred. As the data rate increases, the system becomes more sensitive to variations in dispersion within the signal bandwidth. Accordingly, the linear phase is a crucial parameter in defining the useable effective passband in $40-\mathrm{Gb} / \mathrm{s}$ systems.

Based on optical digital filter theory, a dispersion-compensating interleaver pair is presented. These interleavers have a $0.5-\mathrm{dB}$ passband that exceeds $60 \mathrm{GHz}$ and they support both positive and negative phase response.

\section{Using BiREFRINGENT CRYSTAL TO DESIGN DISPERSION-COMPENSATING INTERLEAVERS}

For a long time, birefringent crystal has been used in designing optical filters; such filters comprise birefringent crystal plates and polarizers. The two basic types of birefringent filters are Lyot-Öhman [10], [11] filters and Šolc [12] filters. Both are based on interference between polarized light, which depends on phase retardation between the components of light polarized parallel to the slow and the fast axes of the crystal. Consequently, birefringent crystal is used as an optical delay line, and a half-wave plate is used to alter the polarization between the delay stages. An optical FIR digital filter can, thus, be made by cascading delay lines and controlling the angle of rotation between half-wave plates. The half-wave plates can also be considered to be rotated to generate required Fourier frequency components.

Fig. 1 depicts the configuration of the interleaver that incorporated the birefringent crystal. At the input and outputs of the interleaver, $\mathrm{YVO}_{4}$ walkoff crystal and a half-wave plate were used to ensure that light that passed through the optical delay cells was polarized in only one direction. Each delay cell includes two 
birefringent crystals, namely $\mathrm{YVO}_{4}$ and rutile $\left(\mathrm{TiO}_{2}\right)$, to compensate for any change in temperature. The lengths of $\mathrm{YVO}_{4}$ and rutile are determined using the following:

$$
\begin{aligned}
\Delta n_{1} L_{1}-\Delta n_{2} L_{2} & =m \lambda_{\text {center }}=\frac{c}{\mathrm{FSR}} \\
\frac{d}{d T}\left(\Delta n_{1} L_{1}-\Delta n_{2} L_{2}\right) & =0 \Rightarrow \Delta n_{1} L_{1} \beta_{1}-\Delta n_{2} L_{2} \beta_{2}
\end{aligned}
$$

with

$$
\beta_{i} \equiv \frac{d \Delta n_{i}}{\Delta n_{i} d T}+\frac{d L_{i}}{L_{i} d T}=\frac{d \lambda_{i}}{\lambda_{i} d T}, \quad i=1,2 .
$$

In (1)-(3), $\Delta n_{1}$ and $\Delta n_{2}$ represent the difference between group indexes of the ordinary and extraordinary axes of $\mathrm{YVO}_{4}$ and rutile, respectively, $c$ is the speed of light, FSR is the free spectral range, $L_{i}$ is the length of the crystal, $\lambda_{\text {center }}$ is the central wavelength in the range of operation wavelengths, $m$ is the order of the birefringent wave plate, and $\beta_{\iota}$ is the normalized variation in the wavelength with temperature. For $\mathrm{YVO}_{4}$ and rutile, the difference between group indexes are 0.2139 and 0.2652 , respectively, and the value of $\beta$ are $-26.54 \times 10^{-6} 1 /{ }^{\circ} \mathrm{C}$ and $-99.06 \times 10^{-6} 1 /{ }^{\circ} \mathrm{C}$, respectively. At the central frequency of $193.5 \mathrm{THz}$, these values of $\beta$ correspond to 5.13 and $19.17 \mathrm{GHz} /{ }^{\circ} \mathrm{C}$, respectively. For a $100-\mathrm{GHz}$ interleaver (whose FSR equals $200 \mathrm{GHz}$ ), solving (1) and (2) yields the lengths of the $\mathrm{YVO}_{4}$ and rutile crystals, which are 9.5697 and $2.0685 \mathrm{~mm}$, respectively. Compensation reduces the variation in the temperature of the delay cell to around $0.056 \mathrm{GHz} /{ }^{\circ} \mathrm{C}$; thus, $99 \%$ of the variation in temperature was compensated for. For a temperature changing from $0{ }^{\circ} \mathrm{C}$ to $65^{\circ} \mathrm{C}$, the compensated delay cell has a central frequency drift of around $3.7 \mathrm{GHz}$.

The perfect interleaver function is a periodic square-wave response in the frequency domain. A square-wave amplitude response has only odd Fourier frequency components. Equations (4) and (5) state the Fourier frequency components generated by $L-2 L$ and $L-3 L$ delay lines, when the FSR of the unit delay cell $L$ equals $\omega$. For a digital filter with only odd Fourier frequency components, the $L-2 L$, and not the $L-3 L$, configuration must be selected

$$
\begin{aligned}
& L-2 L:\left[\begin{array}{c}
\frac{\omega}{2} \\
-\frac{\omega}{2}
\end{array}\right] \times\left[\begin{array}{c}
\omega \\
-\omega
\end{array}\right] \Rightarrow\left[\begin{array}{c}
\frac{3 \omega}{2} \\
\frac{\omega}{2} \\
-\frac{\omega}{2} \\
-\frac{3 \omega}{2}
\end{array}\right] \text { amplitude } \\
& \longrightarrow \\
& \left.\begin{array}{c}
3 \omega \\
\omega \\
-\omega \\
-3 \omega
\end{array}\right] \\
& L-3 L:\left[\begin{array}{c}
\frac{\omega}{2} \\
-\frac{\omega}{2}
\end{array}\right] \times\left[\begin{array}{c}
\frac{3 \omega}{2} \\
-\frac{3 \omega}{2}
\end{array}\right] \Rightarrow\left[\begin{array}{c}
2 \omega \\
\omega \\
-2 \omega \\
-\omega
\end{array}\right] \stackrel{\text { amplitude }}{\longrightarrow}\left[\begin{array}{c}
4 \omega \\
2 \omega \\
-2 \omega \\
-4 \omega
\end{array}\right] .
\end{aligned}
$$

Higher-frequency Fourier components must be included to sharpen the frequency edge response. More delay line stages must be added to increase the highest Fourier frequency; the passband, the insertion loss, the size, the reliability, and the cost are all traded off against each other. For a $100-\mathrm{GHz}$ interleaver, $L-2 L-2 L$ can be used to meet the passband and delay requirements for $40-\mathrm{Gb} / \mathrm{s}$ transmission without difficulty. If only a $10-\mathrm{Gb} / \mathrm{s}$ signal is transmitted, an $L-2 L$ design will suffice. Once

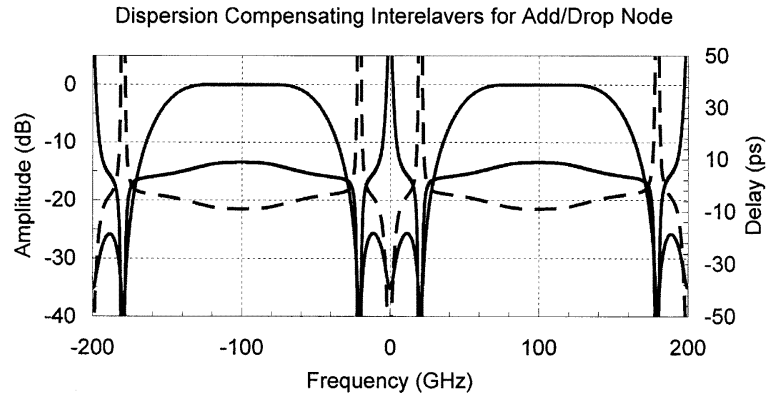

-Mux Amp - DeMux Amp - Mux Delay —DeMux Delay

(a)

Simulation Results of a Cascaded Interelaver Pair

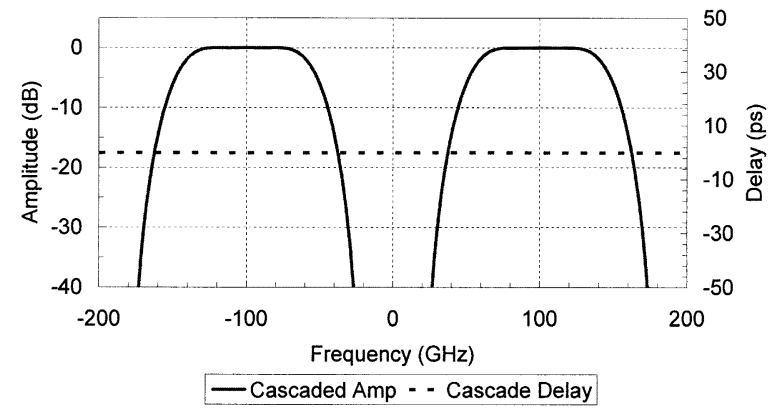

(b)

Fig. 2. (a) Simulated amplitude and delay responses of two types of interleavers. (b) Simulated cascaded amplitude and delay response of a dispersion-compensating pair of interleavers.

the configuration has been selected, the angles of the wave plates must be determined. Optimization tools in standard Matlab or Mathematica programs can be used. The goal is to find the wave plate angles that will minimize

$$
\min \left\{\sum_{n=1}^{N}\left[\hat{x}\left(f_{n}\right)-x\left(f_{n}\right)\right]^{2}\right\}
$$

with

$$
f_{c}-\frac{\mathrm{FSR}}{2} \leq f_{n} \leq f_{c}+\frac{\mathrm{FSR}}{2} .
$$

In (6), $\hat{x}\left(f_{n}\right)$ is the target or ideal interleaver transmission function and $x\left(f_{n}\right)$ is the real transmission function. The transmission function is periodic, so the errors are only summed over one FSR at the central frequency, $f_{c}$. The input polarization angle was shifted by $90^{\circ}$ by changing the position of the half-wave plate at the input walkoff crystal to generate two delay responses.

Fig. 2(a) presents the simulated amplitude and delay response of two types of $L-2 L-2 L$ interleavers. As depicted, changing the input polarization yields two interleavers that have the same amplitude responses, but opposite delay responses. Fig. 2(b) shows the cascaded total amplitude and delay response. As illustrated in this figure, the cascaded interleaver pair will have constant delay and, therefore, a dispersion that is close to zero.

Fig. 3 plots the measured amplitude responses and the delay responses of two types of $100-\mathrm{GHz} L-2 L-2 L$ interleavers. Fig. 3(a) presents the folded transmission responses in a frequency range from 191.8 to $195.5 \mathrm{THz}$. The width of the 

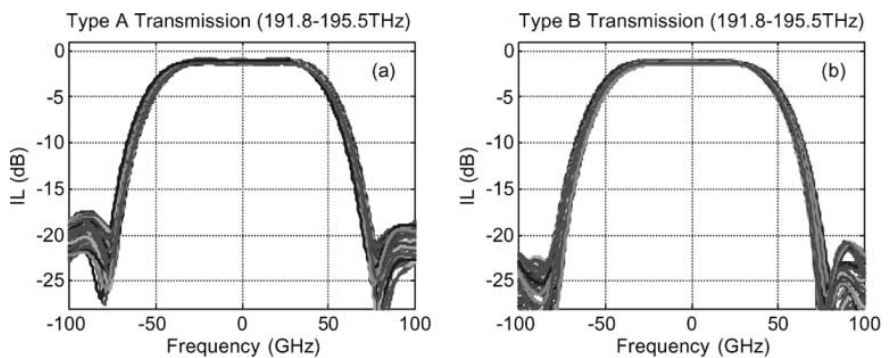

Type A Delay (191.9, 193.9 and 195.3THz)
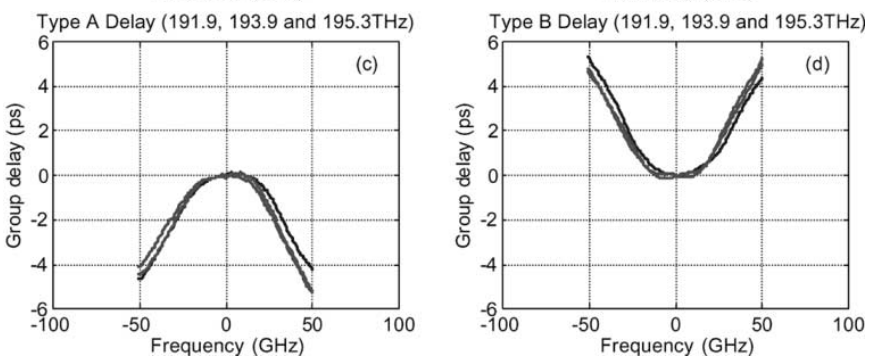

Fig. 3. (a), (b) Measured folded amplitude responses of multiplexer (Mux) and demultiplexer (Demux). (c), (d) Measured folded delay responses of Mux and Demux.

$0.5-\mathrm{dB}$ passband is $60.8 \mathrm{GHz}$ and the insertion loss is $1.45 \mathrm{~dB}$ over all polarizations and temperatures (from $0{ }^{\circ} \mathrm{C}$ to $65^{\circ} \mathrm{C}$ ). As illustrated in Fig. 3(a)-(d), Type A and B interleavers have identical transmission spectra but reversed delay responses. Type A and B interleavers can be cascaded to generate a linear phase interleaver pair with a total dispersion near zero, which characteristic is desirable, particularly in metro add-drop applications and/or high bit rate transmission systems.

A Monte Carlo simulation of manufacturing imprecision was conducted to estimate the design tolerance and the cascaded delay performance. Two parameters were found to dominate the performance - the lengths of the crystals and the angles of the half-wave plates. The variation of the lengths of crystals and the angles of the half-wave plates were assumed to follow a Gaussian distribution with standard deviations $\sigma$ of $0.5 \mu \mathrm{m}$ and $0.6^{\circ}$, respectively. Fig. 4(a) shows the simulation results obtained for ten cascaded interleaver pairs; the total delay is under $0.5 \mathrm{ps}$ within the passband $+/-30 \mathrm{GHz}$. Fig. 4(b) plots the measured results made of seven cascaded interleaver pairs. The total delay is under $1 \mathrm{ps}$ in the passband. Although the recirculating loop simulation and the experiment to estimate the impact on system performance have not been completed, the authors are optimistic that the dispersion will minimally degrade a $40-\mathrm{Gb} / \mathrm{s}$ system.

\section{CONCLUSION}

Dispersion-compensating interleaver pairs for metro add-drop applications have been designed and experimentally demonstrated. Seven pairs of cascaded Type A or B interleavers have a $0.5-\mathrm{dB}$ passband width that is wider than $60 \mathrm{GHz}$, insertion losses of less than $1.5 \mathrm{~dB}$, and maximum group delays of under 1 ps. A total dispersion of around zero considerably increases the effective bandwidth and greatly increases the number of cascadable add-drop nodes.
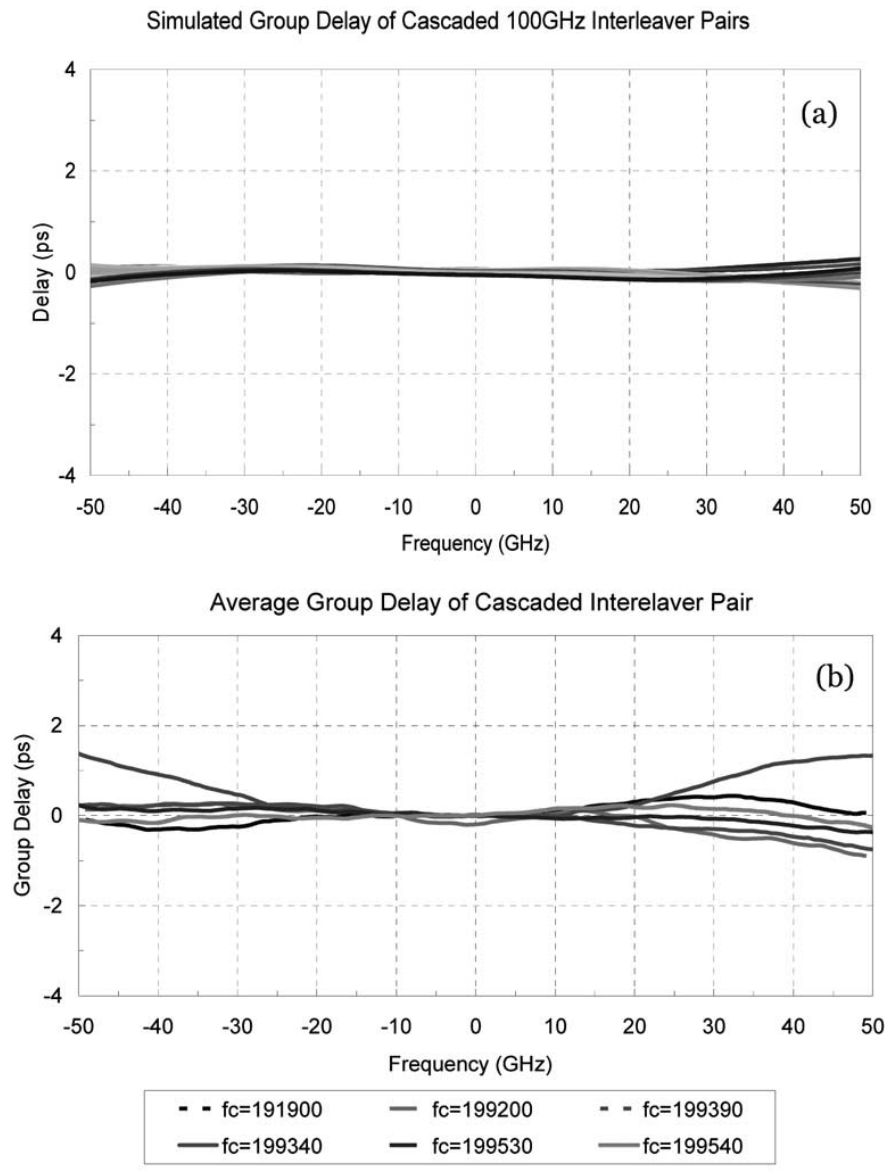

Fig. 4. (a) Simulated cascaded delay responses and (b) measured cascaded delay responses.

\section{REFERENCES}

[1] A. V. Oppenheim, R. W. Schafer, and J. R. Buck, Discrete-Time Signal Processing, 2nd ed. Englewood Cliffs, NJ: Prentice-Hall, 1999.

[2] G. Lenz, B. J. Eggleton, C. R. Giles, C. K. Madsen, and R. E. Slusher, "Dispersive properties of optical filters for WDM systems," IEEE J. Quantum Electron., vol. 34, pp. 1390-1402, Aug. 1998.

[3] C. K. Madsen and J. H. Zhao, Optical Filter Design and Analysis: A Signal Processing Approach. New York: Wiley, 1999.

[4] K. Jinguji and M. Oguma, "Optical half-band filters," J. Lightwave Technol., vol. 18, pp. 252-259, Feb. 2000.

[5] K. Tai, B. Chang, J. Chen, C. Mao, T. Ducellier, J. Xie, L. Mao, and J. Wheeldon, "Wavelength-interleaving bidirectional circulators," IEEE Photon. Technol. Lett., vol. 13, pp. 320-322, Apr. 2001.

[6] K. Tai, K. W. Change, and J. H. Chen, "Interleavers/de-Interleavers Causing Little or no Dispersion of Optical Signals," U.S. Patent 6301046 , Oct. 9, 2001.

[7] B. J. Offrein, R. Germann, G. L. Bona, F. Horst, and H. W. M. Salemink, "Tunable optical add/drop components in silicon-oxynitride waveguide structures," in Eur. Conf. Optical Communications (ECOC'98), 1998, pp. $325-326$.

[8] Y. P. Li, C. H. Henry, E. J. Laskowski, C. Y. Mak, and H. H. Yaffe, “A waveguide EDFA gain equalization filter," Electron. Lett., vol. 31, no. 23, pp. 2005-2006, 1995.

[9] K. Takiguchi, K. Jinguji, and Y. Ohmori, "Variable group-delay dispersion equalizer based on a lattice-form programmable optical filter," Electron. Lett., vol. 31, no. 15, pp. 1240-1241, 1995.

[10] B. Lyot, "Optical apparatus with wide field using interference of polarized light," C. R. Acad. Sci. (Paris), vol. 195, pp. 1593-1597, 1993.

[11] Y. Öhman, "A new monochromator," Nature, vol. 41, pp. 291-296, 1938.

[12] I. Šolc, "Birefringent chain filters," J. Opt. Soc. Amer, vol. 55, pp. 621-624, 1965. 